\title{
Video Article \\ Surface Electromyographic Biofeedback as a Rehabilitation Tool for Patients with Global Brachial Plexus Injury Receiving Bionic Reconstruction
}

\author{
Laura A. Hruby ${ }^{1,2}$, Agnes Sturma ${ }^{1,3}$, Oskar C. Aszmann ${ }^{1,4}$ \\ ${ }^{1}$ Clinical Laboratory for Bionic Extremity Reconstruction, Medical University of Vienna \\ ${ }^{2}$ Department of Orthopaedics and Trauma Surgery, Medical University of Vienna \\ ${ }^{3}$ Department of Bioengineering, Imperial College London \\ ${ }^{4}$ Division of Plastic and Reconstructive Surgery, Department of Surgery, Medical University of Vienna
}

Correspondence to: Agnes Sturma at agnes.sturma@meduniwien.ac.at

URL: https://www.jove.com/video/59839

DOI: doi: $10.3791 / 59839$

Keywords: Neuroscience, Issue 151, surface EMG biofeedback, rehabilitation of nerve injuries, biofeedback, bionic reconstruction, brachial plexus injury, nerve root avulsion, prosthetic rehabilitation, prosthetic reconstruction

Date Published: 9/28/2019

Citation: Hruby, L.A., Sturma, A., Aszmann, O.C. Surface Electromyographic Biofeedback as a Rehabilitation Tool for Patients with Global Brachial Plexus Injury Receiving Bionic Reconstruction. J. Vis. Exp. (151), e59839, doi:10.3791/59839 (2019).

\section{Abstract}

In patients with global brachial plexus injury and lack of biological treatment alternatives, bionic reconstruction, including the elective amputation of the functionless hand and its replacement with a prosthesis, has recently been described. Optimal prosthetic function depends on a structured rehabilitation protocol, as residual muscle activity in a patient's arm is later translated into prosthetic function. Surface electromyographic (sEMG) biofeedback has been used during rehabilitation after stroke, but has so far not been used in patients with complex peripheral nerve injuries. Here, we present our rehabilitation protocol implemented in patients with global brachial plexus injuries suitable for bionic reconstruction, starting from identification of SEMG signals to final prosthetic training. This structured rehabilitation program facilitates motor relearning, which may be a cognitively debilitating process after complex nerve root avulsion injuries, aberrant re-innervation and extra-anatomical reconstruction (as is the case with nerve transfer surgery). The rehabilitation protocol using sEMG biofeedback aids in the establishment of new motor patterns as patients are being made aware of the advancing re-innervation process of target muscles. Additionally, faint signals may also be trained and improved using sEMG biofeedback, rendering a clinically "useless" muscle (exhibiting muscle strength M1 on the British Medical Research Council [BMRC] scale) eligible for dexterous prosthetic hand control. Furthermore, functional outcome scores after successful bionic reconstruction are presented in this article.

\section{Video Link}

The video component of this article can be found at https://www.jove.com/video/59839/

\section{Introduction}

Global brachial plexus injuries including the traumatic avulsion of nerve roots from the spinal cord represent one of the most severe nerve injuries in humans and usually affect young, otherwise healthy patients in the prime of life ${ }^{1,2}$. Depending on the number of nerve roots avulsed, complete upper limb paralysis may ensue since the nerval connection from the brain to the arm and hand is disrupted. Traditionally, the avulsion of nerve roots has been associated with poor outcomes ${ }^{3}$. With microsurgical nerve techniques gaining ground within the last decades, surgical results have been improved and useful motor function in the shoulder and elbow are usually restored ${ }^{4,5}$. The intrinsic musculature in the hand, which lies most distally, typically undergoes fatty degeneration resulting in irreversible atrophy before regenerating axons may reach it ${ }^{6}$. For such cases bionic reconstruction, which includes the elective amputation of the functionless "plexus" hand and its replacement with a mechatronic hand, has been described ${ }^{7,8}$. Residual muscle activity in a patient's forearm, which may be clinically insignificant (isometric contractions, M1 on the British Medical Research Council [BMRC] scale), is picked up from transcutaneous electrodes sensing electromyographic activity, which is then translated into various movements of a prosthetic hand ${ }^{9}$.

Enough surface electromyographic (sEMG) signals may be present upon initial consultation. In some cases, however, additional signals need to be established performing selective nerve and muscle transfers ${ }^{7}$. In either case, a structured rehabilitation protocol is needed to ensure sEMG signal consistency and subsequent optimal prosthetic function at the end of the process. A major challenge following nerve root avulsion and aberrant re-innervation as well as after nerve transfer surgery is the establishment of new motor patterns to allow volitional control over the target muscle. sEMG biofeedback methods have been widely used in the rehabilitation of stroke ${ }^{10}$. This method allows direct visualization of muscular activity that would otherwise be unnoticed due to muscular weakness and/or co-activation of antagonists. It thereby encourages patients to train their weak muscles, while providing precise feedback on the correct execution of motor tasks ${ }^{11}$.

In a recent publication we have shown for the first time that sEMG biofeedback may also be used in the rehabilitation of complex peripheral nerve injuries ${ }^{12}$. We believe that sEMG biofeedback is an extremely useful method to make a patient aware of the advancing re-innervation process after nerve transfer surgery. Also, faint muscle activity, which formerly was of no use to the patient, may be trained and strengthened 
for later prosthetic control using SEMG biofeedback, which allows concrete visualization of otherwise unnoticed muscle activity to both clinician and patient. The training progress may therefore be well comprehended and documented. Additionally, the use of direct feedback on muscle activity allows the clinician to correlate various motor commands with the associated signal amplitude and consistency, establishing the best motor strategies to allow robust prosthetic control in the future. In summary, the goal of this method is to facilitate the rehabilitation process by increasing a patient's understanding, awareness and control of his/her sEMG signals, which will later drive a prosthetic hand.

\section{Protocol}

The clinical implementation of this rehabilitation protocol was approved by the ethics committee of the Medical University of Vienna (ethical vote number: 1009/2014), Austria and carried out in accordance with the standards set by the Declaration of Helsinki. All patients provided written informed consent to participating in this study.

NOTE: Previous publications by Aszmann et al. ${ }^{7}$ and Hruby et al. ${ }^{8,13}$ are available describing the concept, treatment algorithm, and psychosocial prerequisites regarding bionic reconstruction in great detail. Table of Materials references all materials and equipment used in the proposed rehabilitation protocol.

\section{Patient assessment upon initial consultation}

1. For all steps of patient assessment, rehabilitation and training, find an office or examination room, where the patient is alone in a quiet atmosphere without disturbances. Make sure to have enough space to examine the patient and to set up the sEMG biofeedback system

2. Obtain a detailed case history from the patient including injury mechanism and first care, reports on previous nerve repair surgeries, and subjective disability in day-to-day life.

3. Consider only patients for bionic reconstruction with failed biological treatment alternatives (i.e., nerve repair, nerve transfers, secondary reconstructions resulting in futile upper limb function). Exclude patients with simultaneous damage to the central nervous system, unstable fractures of the affected limb, untreated and/or resilient mental health problems, drug addiction, lack of compliance and commitment to adhere to a long-lasting rehabilitation program.

4. Perform a detailed clinical examination focusing on the current upper limb function. Clinically assess the function of all major muscles in the affected arm and hand using the BMRC grading scale.

5. Assess in a multidisciplinary team consisting of reconstructive surgeons, orthopaedic surgeons, physiatrists, psychologists and physiotherapists whether biological treatment alternatives are possible. Explain to the patient that the functionality of a myoelectric prosthesis can by no means compare with that of a biological hand.

6. Ask the patient about his/her motifs and perspectives on bionic reconstruction (see a previous publication ${ }^{13}$ including the structured interview with a psychologist to assess whether a patient is psychosocially fit to go through the process of bionic reconstruction).

7. Assess whether Tinel signs can be elicited along the neural axis of the major peripheral nerves indicating the presence of viable axons suitable for nerve transfer surgery.

8. Apart from the patient assessment, also vaguely outline a possible timeline of the whole process, which depends on the availability of detectable EMG signals. If other interventions, such as psychological support, posture training, and/or strengthening of the remaining muscles are indicated, start them as soon as possible.

\section{Identification of sEMG signals}

1. Set up a system for sEMG biofeedback on a table in a quiet room. This can be a stand-alone device, or one connected to a computer. If a computer is used, connect the EMG device with the computer by plugging in all cables and start the appropriate software on the computer

2. To reduce impedance, prepare the patient's skin by carefully shaving the respective body part and/or by gently removing dead skin cells with a peeling gel or a wet paper towel.

3. Shortly explain the functionality of the EMG device and associated computer software to the patient.

4. Position the patient in front of the computer screen.

5. Ask the patient to think of hand movements and simultaneously attempt to contract the muscles intended to perform a specific action (such as extending the wrist, making a fist, flexing the thumb, etc.), even if this will not result in real movement of his functionless hand. Palpate his/her forearm for (weak) muscle contraction.

6. Place a sEMG electrode on the exact skin position, where muscle contraction can be palpated with the finger, e.g., on the dorsal extensor compartment $5 \mathrm{~cm}$ distal to the elbow joint when asking the patient to think of extending his/her wrist and fingers.

NOTE: While sEMG activity may be detected with wet and dry electrodes, dry electrodes are prepared for testing as these can be easily moved on the skin to check for optimal positions.

7. Repeat the motor command used before (i.e., extension of wrist and fingers) to elicit contraction of the muscle.

1. Observe the EMG signal on the computer screen and see if the amplitude consistently increases when the patient attempts to contract the muscle intended to perform a specific action (i.e., extending the wrist and fingers).

2. If the amplitude is not high enough (less than 2-3 times of the background noise ${ }^{12}$ ) or the signal is inconsistent, try other motor commands with the same electrode position and see if higher amplitudes can be obtained.

8. Repeat the procedure for a different muscle or muscle group. For example, move the sEMG electrode to the volar aspect of the forearm, placing it on the pronator teres muscle, and ask the patient to attempt pronating his/her forearm. Observe the signal on the computer screen and see if the amplitude repeatedly increases when the patient thinks of this movement.

NOTE: In some patients, no muscle activity is palpable. Here, three or more sEMG electrodes should be placed on the volar, dorsal and radial aspect of the forearm and various motor commands should be attempted, closely observing all signals for amplitude changes even with the slightest changes in electrode positioning (see Figure 1). 


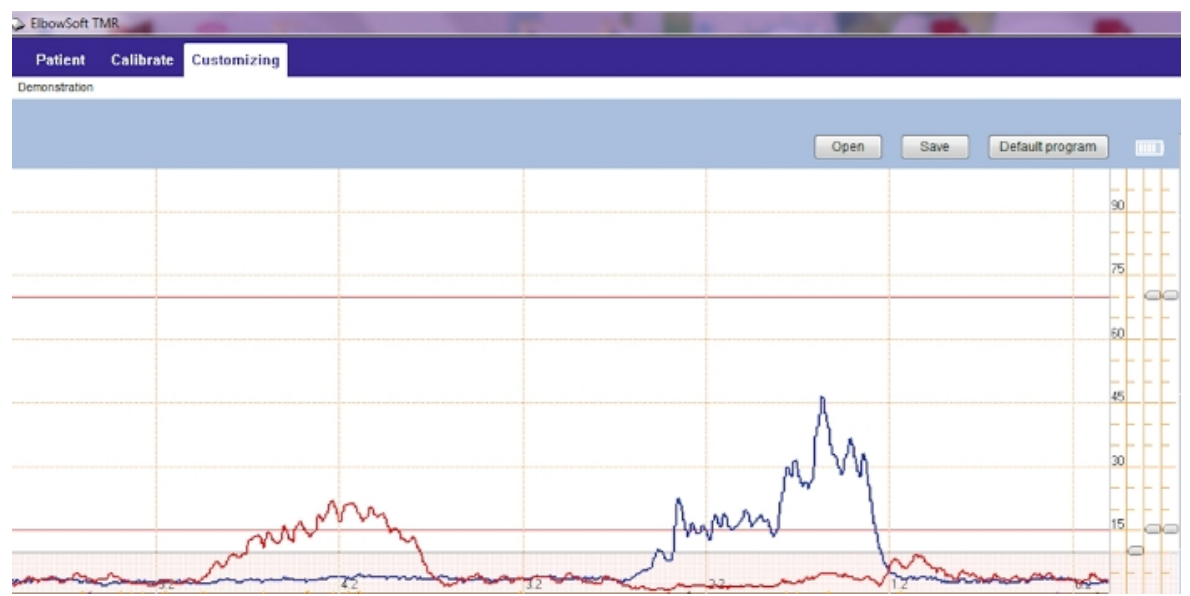

Figure 1: Screenshot of EMG signals on a computer screen.

To identify EMG activity, two or more electrodes can be placed on a patient's forearm asking him/her to attempt various movements. In this specific case, the electrode on the volar aspect of the forearm senses EMG activity as reflected by the first, red wave displayed on the computer screen, when the patient attempts to close his/her hand. Signal separation in this patient is satisfying, since the blue signal, which corresponds to the second electrode placed on the dorsal aspect of the forearm, does not reach the threshold. When the patient thinks of opening the hand, the amplitude of the blue signal exceeds the threshold, while the red signal remains almost inactive. Please click here to view a larger version of this figure.

9. Also try motor commands and electrode positions that differ from the "normal" anatomy as aberrant re-innervation and extra-anatomical reconstructions as in nerve transfers have altered the neural input to the partially denervated muscles.

10. If no muscle activity is found in the forearm, repeat the procedure on the upper arm and shoulder girdle.

NOTE: In some patients, no sEMG signals are found. In these, nerve and muscle transfers need to be performed to establish new EMG signal sites (the detailed surgical concept may be found elsewhere ${ }^{7}$ ), delaying signal training for 6-9 months. For dexterous prosthetic hand control at least two separable EMG signals are needed.

\section{3. sEMG-guided signal training}

NOTE: The training sessions for sEMG-guided signal training should not exceed 30 min as this leads to muscle fatigue, which is hindering successful motor learning. The described steps need to be repeated over an extended period of time to ensure good neuromuscular coordination as needed later for reliable prosthetic control.

1. As soon as two or more EMG signals have been identified, encourage the patient to activate them alternately (see Figure $2 \mathrm{~A}$ ). To reliably drive a prosthesis, the independent EMG signals need to be controlled without interference.

1. Adjust the voltage gain of each signal independently to achieve a similar amplitude threshold for all signals during training, which will make signal separation and comprehension easier for the patient.

2. Repeat and explain to the patient the mechanics of a prosthetic hand: slight muscle contraction will ultimately lead to improved signal separation and must be preferred over muscle strength, i.e., a signal's amplitude. 


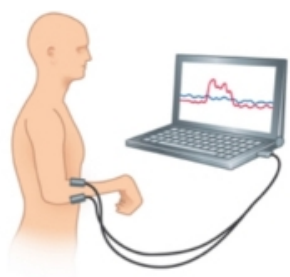

after identification of EMG signals their separation is trained using sEMG biofeedback

C

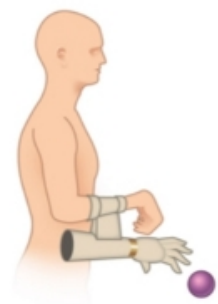

a hybrid prosthetic hand is fitted below the paralyzed hand to allow visualization of

prospective prosthetic hand use

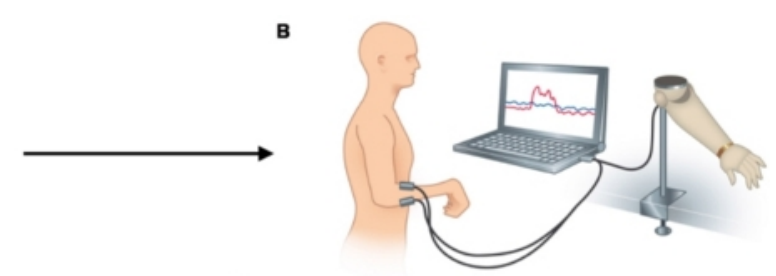

a table top prosthesis to experience and train possible prosthetic function

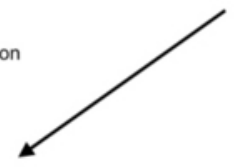

D

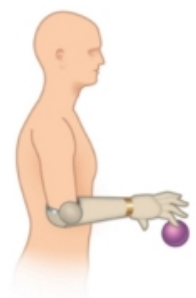

after amputation guided prosthetic training improves reliable prosthetic function

Figure 2: sEMG-guided rehabilitation for patients with bionic hand reconstruction.

(A) With direct visualization of muscle activity, various motor commands may be attempted to identify the highest EMG amplitude over a specific target muscle and different signal positions can be compared. (B) Using a table top prosthesis, the EMG activity in a patient's arm is directly translated into prosthetic function. (C) The fitting of a hybrid prosthetic hand allows the patient to visualize and comprehend future prosthetic hand use. (D) After prosthetic reconstruction, EMG signals can be trained and optimized either with sEMG biofeedback or with the prosthetic hand itself. This figure has been modified from Sturma et al. ${ }^{12}$ and reproduced with permission from Frontiers in Neuroscience. Please click here to view a larger version of this figure.

2. Observe the EMG signals on the computer screen and make the patient aware whether the two signals are co-activated when attempting a specific movement. Explain to the patient that two signals should not be co-activated during the attempt of one specific action, as each EMG signal is linked to a specific prosthetic action. Co-activated signals will therefore not result in the action desired by the patient.

3. Instruct the patient to try different (slight) movements and observe which precise movement patterns are the best ones in regard to signal separation. Encourage the patient to train these movements.

4. Let the patient know that perfect signal separation is unlikely at the beginning of the training but will improve with a high number of repetitions. CAUTION: Allow phases of relaxation as muscle strength may decrease faster in patients with complex nerve injuries and faint myoactivity.

5. With improved signal consistency, instruct the patient to generate a higher signal amplitude to further strengthen the muscle and its signal.

6. With consistent EMG signal separation and solid control, install a table top prosthesis connected to the corresponding EMG software and the electrodes placed on the patient's forearm/arm. This will directly translate EMG activity into mechanical prosthetic function (see Figure 2B and Figure 3). 


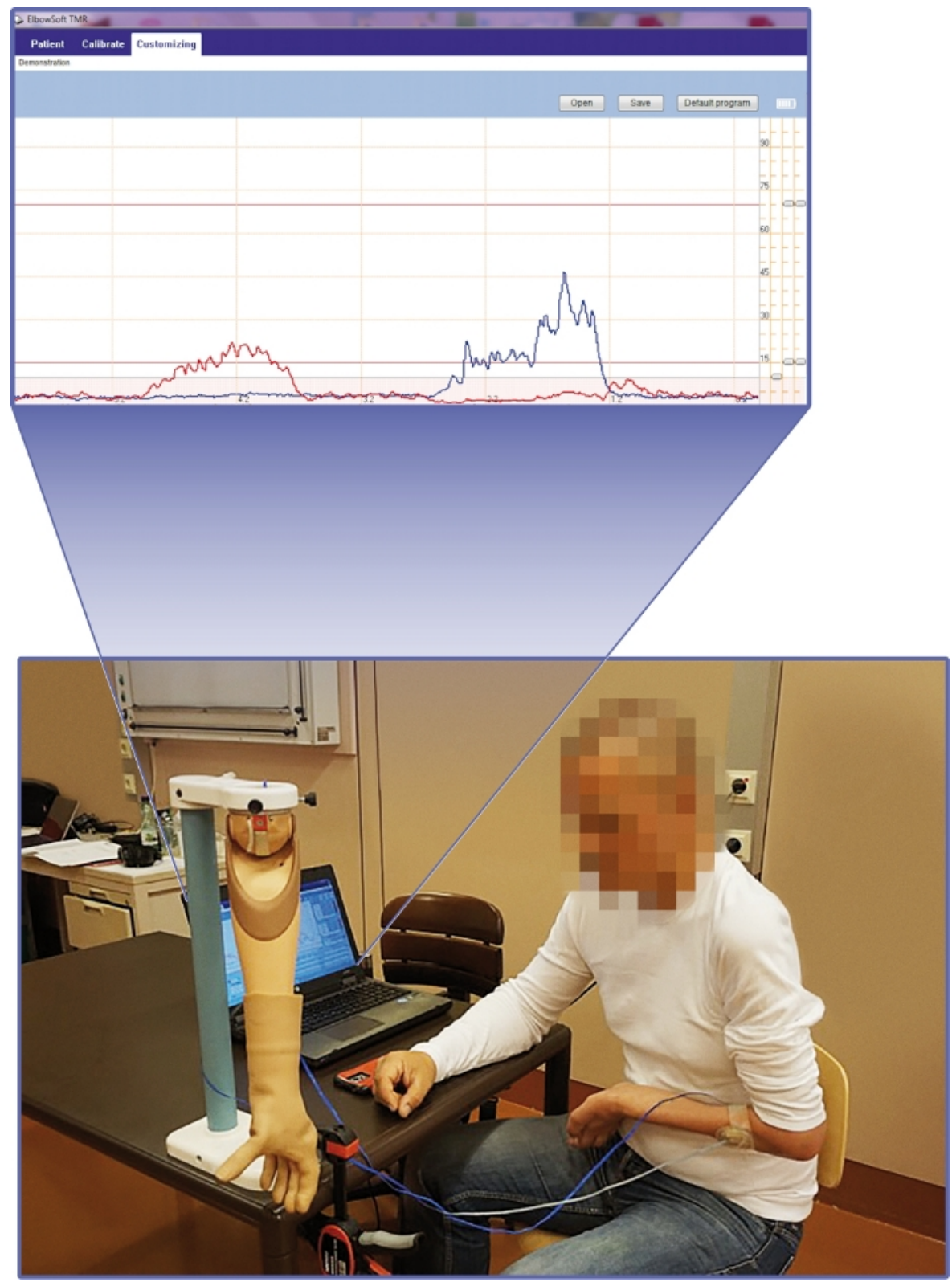

Figure 3: Patient in front of a table top prosthesis and screenshot of his two signals on a computer screen.

On the patient's forearm, two electrodes sense EMG activity. These two signals are displayed as color-coded graphs on the computer screen (red and blue) and are simultaneously translated into prosthetic movement, allowing the patient to comprehend the relationship between signal quality and prosthetic control. Please click here to view a larger version of this figure.

7. Educate the patient that myoelectric prostheses with direct control use the input of one electrode (i.e., the muscular activity detected from one electrode) to control one prosthetic movement.

8. Make the patient aware of the correlation between the signal's appearance (mostly amplitude height) on the computer screen and the speed/ strength of the prosthetic movement, in case a device with proportional control of movement speed is chosen.

NOTE: Depending on the number of available EMG signals and the degrees of freedom of the final prosthetic device, it might be necessary to use methods for switching between these degrees of freedom. One frequently used method of switching between degrees of freedom (e.g., hand open/close to pronation/supination) is via the simultaneous contraction of two muscles, also known as co-contraction ${ }^{14}$.

9. Train co-contraction. Let the patient observe the EMG signals on the computer screen and the prosthesis. If the prosthetic device does not move, i.e., open/close during co-contraction, the patient is doing it correctly.

\section{Hybrid hand fitting and prosthetic training}

1. Mark the electrode positions on the patient's skin, which have been defined as optimal for reliable prosthetic control and let an orthopedic technician manufacture a preliminary prosthetic socket designed with these exact electrode positions.

2. Mount a hybrid prosthetic hand with the individually tailored socket onto or below the functionless "plexus" hand (see Figure 2C).

3. Simultaneously run the EMG software program to make the patient aware of his/her actions. 
4. Alternately train different prosthetic movements. Electrodes may also be placed on adjoining muscles along the upper arm and shoulder girdle to avoid unconscious co-contractions, which will lead to fatigue in the whole upper limb with increased wearing times.

1. Start with simple prosthetic movements (only open/close the hand without any co-contraction) with the weight of the prosthetic device being supported.

2. Move on to simple prosthetic movements in different arm positions, such as the elbow being extended or flexed alternately. Make the patient aware of signal discrepancies when adjusting the various arm positions and train signal consistency in all positions. NOTE: After spontaneous nerve regeneration unintended co-activation of different muscle or muscle groups often occurs due to aberrant re-innervation, which may hamper coordinated movements and disable adequate muscle activities ${ }^{15}$. Faint unintended muscle contraction often occurs when moving the arm, which is sensed by sEMG sensors and translated into prosthetic movement. This may result in poor prosthetic control, if not adequately addressed during rehabilitation using EMG training and muscle strengthening as described below.

3. In case of cumbersome prosthetic control in different arm positions, thoroughly observe the EMG signals on the computer screen and point out to the patient, in what arm position unintended contraction of one or more muscles leads to signal excursion. Train precise activation of EMG signals in positions that the patient can still handle and slowly change the arm position over time.

4. Perform strength training for elbow flexors (and shoulder muscles, if applicable), if co-activation of the muscles used for prosthetic control is observed while lifting the arm. Explain to the patient that a stronger muscle (i.e., a muscle that does not work with its maximum voluntary force during simple lifting tasks) usually also contributes to a better separation of the signals. Also perform strength training, if the upper limb muscles are too weak to move the prosthetic device in three-dimensional space and/or stabilize the shoulder while doing so.

5. Continue with simple grasping tasks, such as picking up little boxes and manipulating small objects (see Figure 2C).

6. Finally, train simple tasks of daily living like opening a door, folding a towel or opening a bottle.

NOTE: Many tasks might be restricted due to the fact that the paralyzed hand gets in the way, and the device might feel rather heavy as the patient has to lift the weight of his/her own hand in addition to the hybrid prosthetic hand.

5. If the signal quality is insufficient, it might be beneficial to go back to signal training on the computer screen. In all tasks specifically look for co-activation of signals on the computer screen and further improve signal independency.

6. Assess upper limb function using the hybrid prosthetic hand and record video of the test results. Additionally, use the same assessments for the paralyzed hand in order to document the functional benefit expected from prosthetic replacement of the functionless hand.

\section{Elective amputation and prosthetic hand replacement}

1. Precisely plan the level of amputation depending on the site of the various EMG signals (transradial, transhumeral or, in rare cases, glenohumeral) in the multidisciplinary team consisting of the patient's physiotherapist/EMG trainer, the surgeon responsible for the amputation and the psychologist familiar with the patient's expectations.

2. Ask the patient if he/she has any unresolved questions regarding the planned amputation and clearly communicate that it is possible at any time prior to amputation to revoke this decision, which will otherwise result in an irreversible and life-altering surgery.

3. Perform the standardized assessment of upper limb function using the functionless hand and video-tape the results

4. Perform the standardized assessment of upper limb function using the hybrid prosthetic hand and video-tape the results to document the benefits of a future prosthetic fitting.

5. Perform the elective amputation of the functionless limb as described previously ${ }^{7,8}$.

6. Allow post-operative wound healing and let the patient train adjacent joints for improved upper limb mobility. After 4-6 weeks, train the EMG signals as described above and define the best hotspots for electrode positions. NOTE: These electrode positions and motor commands might differ slightly from the ones found before amputation.

7. Let an orthopedic technician design the final prosthetic socket using the previously defined EMG electrode positions (see Figure 4, illustrating a possible socket design in one of the included patients).

NOTE: While there is no specific socket design recommended, the exact position of the electrodes and their adhesion to the stump's skin are of utmost importance as brachial plexus patients have a largely reduced neuro-muscular interface. 

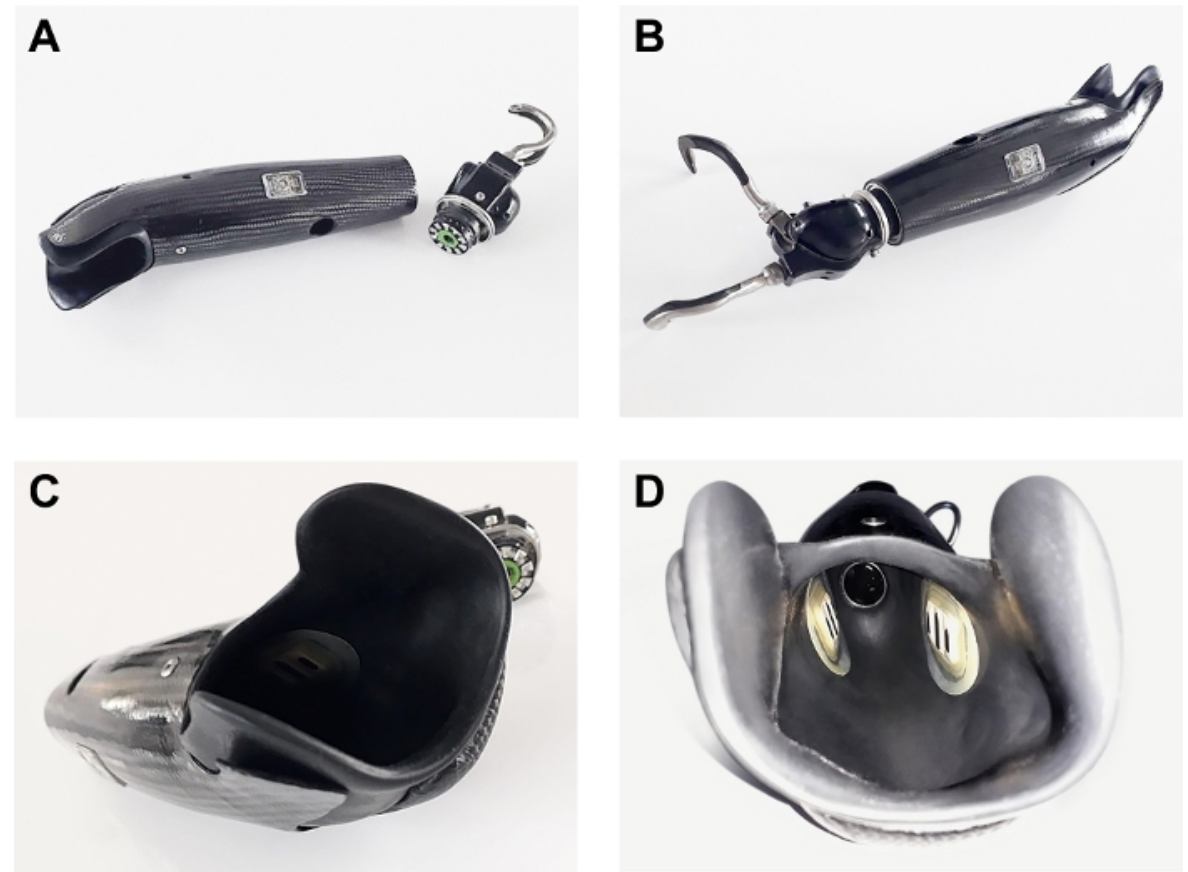

Figure 4: Example of a possible prosthesis and socket design.

(A) This patient's prosthesis consists of an outer sheath made of carbon. (B) Instead of a prosthetic hand, the patient prefers to use a hook, which opens and closes, as a grasping tool. (C,D) The two electrodes are integrated in the prosthesis. The patient wears a silicone liner with two holes in it, allowing direct skin contact with the two electrodes (not shown). Please click here to view a larger version of this figure.

8. Start the prosthetic training.

1. Again, start with simple prosthetic movements (only open/close the hand without any co-contraction) with the weight of the prosthetic device being supported.

2. Move on to simple prosthetic movements in different arm positions, such as the elbow being extended or flexed alternately.

3. Continue with simple grasping tasks such as picking up little boxes and manipulating small objects (see Figure 2D).

4. Finally, train activities of daily living, again starting with rather simple tasks (as opening a door) and slowly add complexity and tasks that the patient considers relevant for his/her specific life situation.

9. Three months after prosthetic fitting, repeat the standardized assessment of upper limb function using the prosthetic hand and record a video of the results.

\section{Representative Results}

In six patients with severe brachial plexus injuries including multiple nerve root avulsions the presented rehabilitation protocol using sEMG biofeedback was successfully implemented. Detailed patient characteristics can be found in Table 1. Figure 2 demonstrates the various phases of the structured rehabilitation protocol and detailed explanations on its implementation.

To demonstrate improvements in hand function before and after bionic reconstruction, a standardized assessment evaluating global upper extremity function was performed at two time points: before elective amputation of the functionless "plexus" hand as well as after successful prosthetic reconstruction and rehabilitation. The Action Research Arm test (ARAT) was originally developed to assess global upper extremity motor function in patients with cognitive impairment of hand control ${ }^{16}$. The standardized approach of Yozbatiran et al. ${ }^{17}$ was used in our studies. The ARAT consists of four different sections, which include tasks close to daily living. The test is timed by the observer who also rates the task performance from $0-3$, with 3 indicating normal function. A maximum of 57 points is attainable indicating unimpaired motor function ${ }^{16}$. The number of therapy sessions with sEMG biofeedback and detailed results for each patient can be found in Table 2.

Although patient satisfaction with the offered rehabilitation protocol using sEMG biofeedback was not directly measured, all six patients reported to find it extremely helpful in comprehending the re-innervation process following nerve transfer surgery and to train the contraction of muscles with very faint activity that formerly was of no clinical use to them. 


\begin{tabular}{|c|c|c|c|c|}
\hline Case number & Sex, age (years) & Type of accident & Type of lesion & $\begin{array}{l}\text { Surgeries to improve biotechnological } \\
\text { interface after initial reconstructions have } \\
\text { failed to improve hand function }\end{array}$ \\
\hline 1 & m, 32 & Fall from height & $\begin{array}{l}\text { Avulsion of } \mathrm{C} 7-\mathrm{T} 1 \\
\text { traction injury of the } \\
\text { infraclavicular plexus }\end{array}$ & Elective amputation of the forearm \\
\hline 2 & $\mathrm{~m}, 32$ & Motorcycle accident & $\begin{array}{l}\text { Rupture of all } 3 \text { trunci } \\
\text { of the BP }\end{array}$ & $\begin{array}{l}\text { Free gracilis muscle transferred to forearm } \\
\text { extensor compartment \& neurotization of deep } \\
\text { branch of radial nerve to obturator nerve; } \\
\text { elective amputation of the forearm }\end{array}$ \\
\hline 3 & $\mathrm{~m}, 55$ & Motorcycle accident & Avulsion of C5-T1 & Elective amputation of the upper arm \\
\hline 4 & m, 38 & Motorcycle accident & $\begin{array}{l}\text { Extensive damage to } \\
\text { roots C5-C8; avulsion } \\
\text { of T1 }\end{array}$ & Elective amputation of the forearm \\
\hline 5 & $\mathrm{~m}, 27$ & Motorcycle accident & Avulsion $\mathrm{C} 8-\mathrm{T} 1$ & Elective amputation of the forearm \\
\hline 6 & $m, 43$ & Motorcycle accident & Avulsion of $\mathrm{C} 6-\mathrm{T} 1$ & $\begin{array}{l}\text { Transfer of triceps muscle to infraspinatous } \\
\text { fossa and transfer of biceps muscle to } \\
\text { supraclavicular fossa to improve prosthetic } \\
\text { fitting; Elective amputation of the arm (shoulder } \\
\text { exarticulation) }\end{array}$ \\
\hline
\end{tabular}

Table 1: Patient characteristics. In all patients, bionic reconstruction was initiated due to infeasibility of biological treatment alternatives. Surgeries to establish EMG signals in the fore- and upper arm may include selective nerve and muscle transfers, which will then drive a myoelectric prosthetic hand. Elective amputation is either performed at a transradial or transhumeral level, depending on the residual muscle activity. All selective nerve transfers performed in this patient group were successful. This table has been modified from Sturma et al. ${ }^{12}$ and reproduced with permission from Frontiers in Neuroscience.

\begin{tabular}{|c|c|c|c|c|}
\hline Case number & ARAT at baseline & ARAT at follow-up & Start of sEMG training & $\begin{array}{l}\text { Number of therapy } \\
\text { sessions in total }(30 \\
\text { min each) }\end{array}$ \\
\hline 1 & 7 & 35 & Immediately after first consultation & 24 \\
\hline 2 & 0 & 15 & $\begin{array}{l}\text { Training with one signal immediately after first } \\
\text { consultation; second signal was available } 9 \\
\text { months after free gracillis transfer + nerve } \\
\text { transfer }\end{array}$ & 30 \\
\hline 3 & 0 & 19 & Immediately after first consultation & 16 \\
\hline 4 & 1 & 22 & Immediately after first consultation & 20 \\
\hline 5 & 9 & 42 & $\begin{array}{l}\text { Immediately after decision to aim for a bionic } \\
\text { reconstruction as biologic reconstruction failed }\end{array}$ & 20 \\
\hline 6 & 0 & 17 & Immediately after first consultation & 22 \\
\hline Mean ( \pm SD) & $2.83 \pm 4.07$ & $25.00 \pm 10.94$ & & $22 \pm 4.32$ \\
\hline
\end{tabular}

Table 2: ARAT scores and number of therapy sessions. In the Action Research Arm test (ARAT), patients initially showed negligible upper limb function (mean 2.83, of a maximum of 57 points attainable). Useful function was restored after bionic reconstruction (mean 25.00 , of 57 ). This table has been modified from Sturma et al. ${ }^{12}$ and reproduced with permission from Frontiers in Neuroscience.

\section{Discussion}

Biofeedback approaches have been widely used in the rehabilitation of several neuromuscular disorders, ranging from (hemi)-plegic conditions resulting from central pathologies such as brain hemorrhage and stroke ${ }^{18,19}$ to various musculoskeletal degeneration or injury and their surgical therapy ${ }^{20,21,22}$. Interestingly, the concept of structured biofeedback has not been implemented in clinical practice for peripheral nerve injuries. However, precisely in the rehabilitation of complex nerve injuries, practice, repetition, and structured training programs with appropriate biofeedback are necessary to establish correct motor patterns ${ }^{23}$.

Here, and in a previous study ${ }^{12}$, we presented a structured rehabilitation protocol using sEMG biofeedback for patients with lack of biological treatment alternatives eligible for prosthetic hand replacement, a concept today known as bionic reconstruction. The most apparent advantage of using a sEMG biofeedback set-up in the context of bionic reconstruction arises from the exact definition of sEMG hotspots, i.e., skin locations, where a relatively high amplitude of EMG activity can be measured transcutaneously. Various motor commands may be attempted alternately, as the sensors can easily be moved along the entire forearm, and - in case of missing detectable muscle function in the forearm - also in the upper arm and shoulder girdle. When a patient is asked to attempt to contract the muscles intended to perform a specific action (such as extending the wrist), an electrode can be placed, where (weak) muscle contraction is palpated by the examiner. Observing the EMG signal on the computer screen, one can easily determine whether the signal's amplitude consistently increases, when the patient attempts to contract this muscle. If the 
amplitude is not high enough or the signal is inconsistent, other motor commands with the same electrode position may be attempted. As oppose to needle EMG, this procedure is non-invasive, not painful and can be repeated for all muscles/muscle groups in the arm. Testing various motor commands at different muscle locations allows to identify the EMG hotspots, with the highest amplitude and reproducible activity associated with a specific motor action. After identification of the strongest EMG signals, these may be trained using sEMG biofeedback with regards to signal separation (co-activation of two or more EMG signals must not occur on the computer screen), signal strength (reflected by the EMG signal's amplitude on the computer screen) and signal reproducibility (each attempt to contract the muscle must lead to an excursion of the respective EMG signal). At a later stage of training, EMG activity is directly translated into prosthetic function, first using a table top prosthesis (see Figure 3), which gives additional feedback to the patient allowing fine-tuning of grip strength, and then wearing the physical prosthesis.

In conventional amputees, a vast amount of literature has shown that targeted-muscle-reinnervation (TMR), i.e., the surgical transfer of residual arm nerves to alternative muscle sites in the chest and upper-arm, improves prosthetic function, since these re-innervated muscles serve as biological amplifiers of intuitive motor commands and provide physiologically appropriate EMG signals for prosthetic hand, wrist and elbow control $^{24,25,26,27}$. Using pattern-recognition control systems, EMG data extracted from numerous sEMG signals placed over the skin of these reinnervated muscles can be decoded and translated to specific, reproducible motor outputs, which provides more reliable myoelectric prosthesis control $^{28,29,30}$. Because the number of EMG signal sites and the myoelectric activity of the muscles in patients with brachial plexus avulsion injury are very limited, pattern recognition algorithms may not be used as is done for conventional amputees ${ }^{8}$. Still, with further research and improved technology, these systems may be able to extract more information on the existing faint muscle signals and therefore improve prosthetic function also in this peculiar patient group.

While the presented protocol is considered a guideline, details need to be adapted depending on the patient and the available equipment. Due to aberrant re-innervation occurring after such nerve injuries, motor commands do not necessarily result in the activation of anatomically "correct" muscles ${ }^{12}$. For example, the authors observed EMG activity at the forearm flexor compartment, while patients were attempting to open their hand. Therefore, various motor commands should be tested in order to identify EMG signals. Additionally, the residual muscular function (although in all cases too weak to generate useful hand movements) might largely vary across patients and cause variations in the required training time as shown in Table 2. Further, the choice of the prosthetic device and the number of electrodes used for control change the requirements for the precision of signal separation, the signal amplitude and the need of co-contraction. All of this needs to be taken into account during signal training, hybrid prosthesis training and actual prosthetic training, as it is also recommended in standard prosthetic training of amputees ${ }^{31}$. Regarding the devices used for sEMG biofeedback training, the authors consider devices suitable if they can simultaneously display the number of signals needed for prosthetic control, give real-time feedback, and can be either connected to a computer or display the signals on a screen themselves. Devices that allow adjusting the signal gain during training are preferred.

After rehabilitation, all patients were able to use their prosthesis during daily life activities and were satisfied with the decision to have their functionless hand replaced with a prosthetic device ${ }^{12}$. This functional improvement was reflected by significant increases in the mean ARAT scores from $2.83 \pm 4.07$ to $25.00 \pm 10.94(p=0.028)$.

From our perspective, sEMG biofeedback set-ups present valuable tools to facilitate the cognitively demanding process of motor recovery associated with nerve injury and bionic reconstruction. The identification of optimal EMG electrode positioning and the testing of various motor commands with direct visualization of muscle activity is greatly simplified using sEMG biofeedback in a clinical set-up. Although sEMG biofeedback may also be used in the rehabilitation of biological upper limb function ${ }^{10,12}$, its application in the process of bionic reconstruction is considered particularly effective. Most importantly, the sEMG signals activated during training later reflect the electrode positions within the prosthetic socket, which is individually customized for each patient. Therefore, repetitive activation of these signals during training most likely increases future prosthetic handling and manual capacity. Direct visualization of this muscle activity also allows a patient to comprehend the concept of myoelectric hand control and he/she may follow the training progress more consciously.

In the future, our presented rehabilitation protocol might be extended with more advanced tools to enhance functional outcomes. This might include high density SEMG recordings to facilitate the process of electrode placement via activation heat maps ${ }^{32}$, further virtual solutions to evaluate EMG activity ${ }^{30,33}$, and serious games to enhance training motivation ${ }^{34}$. Additionally, novel technologies for prosthetic control, such as pattern recognition algorithms might also be used ${ }^{28,30,35}$. However, due to the reduced neuro-muscular interface, it is not clear whether currently commercially available systems designed for otherwise healthy amputees would significantly improve prosthetic function in this specific patient group. Future studies should evaluate the applicability and benefits of the listed novel technologies for the rehabilitation of patients with severe brachial plexus injuries. Additionally, controlled trials with higher patient numbers will also allow to demonstrate the positive effects of the current protocol using sEMG biofeedback with a higher level of evidence.

\section{Disclosures}

The authors have nothing to disclose.

\section{Acknowledgments}

This study was funded by the Christian Doppler Research Foundation of the Austrian Council for Research and Technology Development and the Austrian Federal Ministry of Science, Research and Economy. We are grateful to Aron Cserveny for preparation of the illustrations included in the manuscript and to Frontiers in Neuroscience for permission of reproducing the data presented in the original article ${ }^{12}$.

1. Bertelli, J. A., Ghizoni, M. F. Results and current approach for Brachial Plexus reconstruction. Journal of Brachial Plexus and Peripheral Nerve Injury. 6 (1), 2 (2011).

2. Birch, R. Traction lesions of the brachial plexus. British Journal of Hospital Medicine. 32 (3), 140-143 (1984). 
3. Narakas, A. O. The treatment of brachial plexus injuries. International Orthopaedics. 9 (1), $29-36$ (1985).

4. Terzis, J. K., Barbitsioti, A. Primary restoration of elbow flexion in adult post-traumatic plexopathy patients. Journal of Plastic, Reconstructive \& Aesthetic Surgery. 65 (1), 72-84 (2012).

5. Tung, T. H., Mackinnon, S. E. Nerve Transfers: Indications, Techniques, and Outcomes. The Journal of Hand Surgery. 35 (2), $332-341$ (2010).

6. Terzis, J. K., Vekris, M. D., Soucacos, P. N. Brachial plexus root avulsions. World Journal of Surgery. 25 (8), 1049-1061 (2001).

7. Aszmann, O. C. et al. Bionic reconstruction to restore hand function after brachial plexus injury: a case series of three patients. Lancet. 385 (9983), 2183-219 (2015).

8. Hruby, L. A. et al. Algorithm for bionic hand reconstruction in patients with global brachial plexopathies. Journal of Neurosurgery. 127 (5), 1163-1171 (2017)

9. Bergmeister, K. D. et al. Broadband Prosthetic Interfaces: Combining Nerve Transfers and Implantable Multichannel EMG Technology to Decode Spinal Motor Neuron Activity. Frontiers in Neuroscience. 11, 421 (2017).

10. Kim, J. H. The effects of training using EMG biofeedback on stroke patients upper extremity functions. Journal of Physical Therapy Science. 29 (6), 1085-1088 (2017).

11. Merletti, R.P.P. Electromyography: Physiology, Engineering, and Non-Invasive Applications. Wiley IEEE-Press Verlag. (2004).

12. Sturma, A., Hruby, L. A., Prahm, C., Mayer, J. A., Aszmann, O. C. Rehabilitation of Upper Extremity Nerve Injuries Using Surface EMG Biofeedback: Protocols for Clinical Application. Frontiers in Neuroscience. 12, 906 (2018).

13. Hruby, L. A., Pittermann, A., Sturma, A., Aszmann, O. C. The Vienna psychosocial assessment procedure for bionic reconstruction in patients with global brachial plexus injuries. PLoS ONE. 13 (1), e0189592 (2018).

14. Vujaklija, I., Farina, D., Aszmann, O. New developments in prosthetic arm systems. Orthopedic Research and Reviews. 8, 31-39 (2016).

15. Shin, Y. B., Shin, M. J., Chang, J. H., Cha, Y. S., Ko, H. Y. Effects of Botulinum Toxin on Reducing the Co-contraction of Antagonists in Birth Brachial Plexus Palsy. Annals of Rehabilitation Medicine. 38 (1), 127-131 (2014).

16. Lyle, R. C. A performance test for assessment of upper limb function in physical rehabilitation treatment and research. International Journal of Rehabilitation Research. 4 (4), 483-92 (1981).

17. Yozbatiran, N., Der-Yeghiaian, L., Cramer, S. C. A standardized approach to performing the action research arm test. Neurorehabilitation and Neural Repair. 22 (1), 78-90 (2008)

18. Giggins, O. M., Persson, U. M., Caulfield, B. Biofeedback in rehabilitation. Journal of NeuroEngineering and Rehabilitation. 10, 60 (2013).

19. Rayegani, S. M. et al. Effect of neurofeedback and electromyographic-biofeedback therapy on improving hand function in stroke patients. Topics in Stroke Rehabilitation. 21 (2), 137-151 (2014).

20. Pfeufer, D. et al. Training with biofeedback devices improves clinical outcome compared to usual care in patients with unilateral TKA: a systematic review. Knee Surgery, Sports Traumatology, Arthroscopy. 27 (5), 1611-1620 (2018).

21. Huang, H., Lin JJ, Guo, YL, Wang WTJ, Chen YJ. EMG biofeedback effectiveness to alter muscle activity pattern and scapular kinematics in subjects with and without shoulder impingement. Journal of Electromyography and Kinesiology. 23 (1), 267-274 (2013).

22. Oravitan, M., Avram, C. The effectiveness of electromyographic biofeedback as part of a meniscal repair rehabilitation programme. Journal of Sports Science and Medicine. 12 (3), 526-32 (2013).

23. Novak, C. B., von der Heyde, R. L. Evidence and techniques in rehabilitation following nerve injuries. Hand Clinics. 29 (3), $383-392$ (2013)

24. Dumanian, G. A. et al. Targeted reinnervation for transhumeral amputees: current surgical technique and update on results. Plastic and Reconstructive Surgery. 124 (3), 863-869 (2009).

25. Kuiken, T. A. et al. Targeted muscle reinnervation for real-time myoelectric control of multifunction artificial arms. JAMA. 301 (6), 619-628 (2009).

26. Miller, L. A. et al. Control of a six degree of freedom prosthetic arm after targeted muscle reinnervation surgery. Archives of Physical Medicine and Rehabilitation. 89 (11), 2057-2065 (2008).

27. Kuiken, T. A. et al. Targeted reinnervation for enhanced prosthetic arm function in a woman with a proximal amputation: a case study. Lancet. 369 (9559), 371-380 (2007).

28. Scheme, E., Englehart, K. Electromyogram pattern recognition for control of powered upper-limb prostheses: state of the art and challenges for clinical use. Journal of Rehabilitation Research \& Development. 48 (6), 643-659 (2011).

29. Simon, A.M., Lock, B., Stubblefield, K.A. Patient training for functional use of pattern recognition-controlled prostheses. Journal of Prosthetics and Orthotics. 24 (2), 56-64 (2012).

30. Simon, A. M., Hargrove, L. J., Lock, B. A., Kuiken, T. A. Target Achievement Control Test: evaluating real-time myoelectric pattern-recognition control of multifunctional upper-limb prostheses. Journal of Rehabilitation Research \& Development. 48 (6), 619-627 (2011).

31. Johnson, S. S., Mansfield, E. Prosthetic training: upper limb. Physical Medicine and Rehabilitation Clinics of North America. 25 (1), $133-151$ (2014).

32. Kapelner, T. et al. Motor Unit Characteristics after Targeted Muscle Reinnervation. PLoS ONE. 11 (2), e0149772 (2016).

33. Sturma, A. et al. A surface EMG test tool to measure proportional prosthetic control. Biomedizinische Technik. Biomedical Engineering. 60 (3), 207-213 (2015)

34. Prahm, C., Kayali, F., Sturma, A., Aszmann, O. PlayBionic: Game-Based Interventions to Encourage Patient Engagement and Performance in Prosthetic Motor Rehabilitation. PM\&R. 10 (11), 1252-1260 (2018).

35. Roche, A. D. et al. A Structured Rehabilitation Protocol for Improved Multifunctional Prosthetic Control: A Case Study. Journal of Visualized Experiments. (105), e52968 (2015). 